\title{
ANALYSIS OF THE IMPACT OF SPECIAL CONSTRUCTIONS OF GAP SEALS ON THE DYNAMICS OF CENTRIFUGAL MACHINES
}

\author{
Serhii Shevchenko ${ }^{1}$ \\ ${ }^{1}$ Department of Energy Modeling, Institute for Modeling Problems in Power Engineering G. E. Pukhova NAS of Ukraine, Kyiv, Ukraine \\ s.shevchenko@united.productions \\ ORCID: http://orcid.org/0000-0002-5425-9259
}

ARTICLE INFO

Article history:

Received date 10.10.2020

Accepted date 19.10.2020

Published date 30.10 .2020

Section:

Machine building and metal processing

DO I

$10.21303 / 2313-8416.2020 .001485$

KEYWORDS

gap seals

floating rings

deformable seals

labyrinth seals

rotor dynamics
ABS TRACT

The object of research: the influence of the design features of special gap seals on the dynamic characteristics of centrifugal machines.

Investigated problem: harmonization of the consumption and dynamic characteristics of the seals of the rotors of high-speed machines by applying special design methods.

Main scientific results: The mechanism and operating conditions of seals with floating rings are described. It is determined that the most effective from the point of view of dynamic characteristics is the variant of the semi-movable ring. Expressions for determining the conditions of angular and radial immobility of a floating ring are obtained. The design options for deformable gap seals, including deformable floating rings, deformable interwheel seals and seals with an axially movable deformable sleeve, have been investigated. The scope of their application for unique machines with high requirements for tightness and vibration reliability has been determined. Variants of the design of labyrinth seals have been investigated. The analysis shows that the dynamic properties of labyrinth seals significantly depend on the relative position of the ridges. Overlapping ridged seals have the worst dynamic performance of the labyrinth seal designs. The honeycomb seal has more favorable dynamic properties, and the well seal has the best combination of consumption and dynamic characteristics. The dynamic characteristics are especially important for the seals of high-speed rotors of centrifugal machines.

The area of practical use of the research results: Enterprises that manufacture centrifugal machines: pumps and compressors.

Innovative technological product: a methodology for the selection, design and calculation of special designs of gap seals with optimization of dynamic and flow characteristics.

Scope of application of the innovative technological product: The obtained research results will be useful in the design and manufacture of centrifugal pumps and compressors for any parameters.

(C) The Author(s) 2020. This is an open access article under the CC BY license http://creativecommons.org/licenses/by/4.0).

\section{Introduction}

Gap seals according to the accepted classification [1] belong to the class of non-contact. This means that during operation a guaranteed gap is maintained between their sealing surfaces.

Gap throttles separate the cavities in which the medium to be sealed is under different pressures. The flow rate is limited due to the expenditure of potential pressure energy to overcome local resistances, frictional resistance along the channel length, and sometimes inertial resistance. The greater these costs, the smaller the fraction of potential energy is converted into kinetic energy of the flow, the lower the average fluid velocity in the channel and its flow rate. Thus, gap seals do not completely eliminate, but only limit the flow.

\section{1. The object of research}

The object of research is special designs of gap seals and the influence of their design features on the dynamic characteristics of rotary machines.

\section{2. Problem description}

Gap seals work conditions and the functions assigned to them are very diverse. In addition to centrifugal pumps and compressors, they are widely used in hydraulic and pneumatic automa- 
tion systems, in hydraulic and pneumatic drives (hydraulic cylinders, plungers, spools, adjustable throttles, valves), in devices with an unsealed piston, in shut-off, control and safety valves, in various piston machines (piston rings), in hydraulic, steam and gas turbines (labyrinth seals). Gap throttles are available as separate accessories for most other seal types. Radial and end gap throttles are integral elements of hydrostatic and gas support and thrust bearings, rotor axial balancing systems. In all these designs, both the consumption and the dynamic characteristics of the seals are of great importance [2].

The dynamic performance is especially important for high speed rotors. Gap seals, in addition to the function of sealing, perform an equally important function - to improve the vibration state of the rotor. Unfortunately, these functions, as a rule, are in mutual contradiction: constructive measures aimed at increasing the hydraulic resistance, most often worsen the dynamic qualities of seals, their hydrostatic stiffness and damping [3]. The efforts of the designers are aimed at harmonizing these functions.

\section{3. Suggested solution to the problem}

In the process of creating centrifugal pumps for any parameters, in addition to developing an economical flow path, the primary tasks are to reduce vibrations, ensure the required reliability and durability of supports and drive couplings, and develop reliable and sufficiently tight seals. The peculiarity of centrifugal machines is that the listed tasks are interrelated and in most cases can be satisfactorily solved due to the correct choice of the design of the gap seals.

\section{Materials and Methods}

The energy of volumetric losses can be converted into useful energy if the gap seals are used simultaneously as hydrostatic bearings, capable of not only having high radial stiffness, but also effectively damping rotor vibrations. In this case, the energy of the leaks can not only provide the necessary bearing capacity of the supports, but also, most importantly, reduce the rotor vibration to an acceptable level even in the presence of significant imbalance [4].

The influence of the medium is especially significant in the presence of large gradients of velocities and pressures. Such conditions are just typical for small gaps of gap seals, at which large pressure drops are throttled, and one of the walls belongs to a rotating and vibrating rotor [5].

Thus, when choosing the design of gap seals, one must take into account not only their direct purpose - to reduce volumetric losses, but also their equally important function - to provide the necessary vibration characteristics of the rotor [6].

\section{Results}

Let's consider some of the results achieved in this direction in relation to the seals of rotors of centrifugal machines.

\section{1. Floating ring seals}

The floating ring seal [7] (Fig. 1) is a combination of an O-ring B (non-contacting gap seal) and an end contact $C$, which acts as a mechanical end seal. The throttle $B$ is formed by the surface of the rotating shaft 1 and the inner cylindrical surface of the floating ring 4 . The preliminary compression of the ring to the bearing surface of the cover 2 is carried out by elastic elements (springs) 5 located in the sleeve 6 fixed in the housing 7.

Due to the sealed pressure $p_{1}$, an axial force $F_{c}=A_{e} p_{1}$ is created on the loading surface $A_{e}$, which ensures the required density of the end contact $\mathrm{C}$. The radial hydrodynamic force $F_{y}$ arising in the gap seal $\mathrm{B}$ and proportional to the eccentricity of the ring relative to the shaft contributes to the self-centering of the ring if it exceeds friction force $R_{c} \cong f F_{c}$ in the end contact.

Due to the ability of the floating ring to self-centering, the radial clearance in the annular throttle can be made several times smaller than in conventional gap seals, without fear of contact of the ring with the rotating shaft and possible scoring. Because the flow through the throttle ring is proportional to the cube of the gap $H^{3}$ for laminar flow and $H^{3 / 2}$ for turbulent flow, floating ring seals are able to provide a significant reduction in leakage compared to conventional gap seals. 


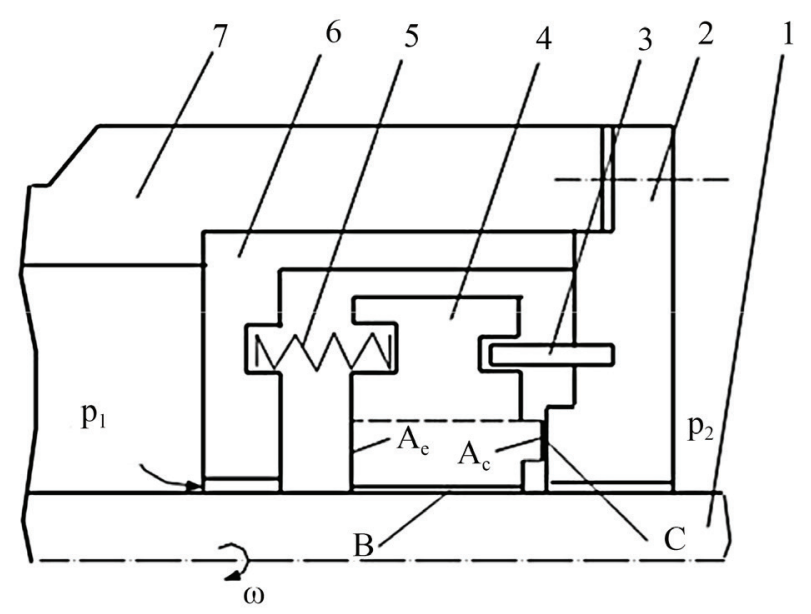

Fig. 1. Diagram of a gap seal with a floating ring

The tightness of the end joint $\mathrm{C}$ depends on the ratio of the contact pressure $p_{c}=F_{c} / A_{c}$ to the sealed pressure $\mathrm{p}_{1}$. The contact pressure, in turn, is determined by the load factor. When there is no back pressure $p_{2}=0, p_{c}=k p_{1}, k=A_{e} / A_{c}$. The design of the seal allows this coefficient to be varied within a wide range, satisfying the requirements of tightness. The floating ring does not rotate; therefore, the frictional power loss in the end joint is an order of magnitude less than in mechanical end seals. This eliminates the problem of heat removal from the contact surfaces, and the seal itself does not have severe restrictions on the circumferential speed of the shaft $v=\omega r$ and on the sealed pressure $p_{1}$. In other words, the factor $p_{1} v$ for floating ring gap seals is not decisive.

It follows from the mechanism of the seal that the ring floats if the self-centering condition is met, i. e. $F_{y \max }>R_{c}$. However, if this condition is not met and the ring does not float, then it is still displaced in the radial direction under the action of impacts from the side of the shaft. Bearing in mind that the impact energy is limited by the work of the friction force at the end joint, it is possible to select materials for which light episodic impacts are not dangerous. The ring relatively easily takes a neutral position, in which shockless operation is ensured. It is only necessary that the amplitude of the lateral vibrations of the shaft does not exceed the value of the radial clearance, and the material of the sealing surfaces resists scuffing during short-term contacts in transient modes. In many cases, such semi-movable or conditionally movable rings turn out to be more effective, since they have increased static and dynamic stability [1].

In real structures, floating rings do not always have the ability to float, but this does not mean that they are inoperative. For comparison, consider the possible modes of operation of the simplest design of a floating ring.

During operation, the floating ring is acted upon by hydrodynamic forces and moments arising in the gap seal and changing in magnitude and direction due to the radial and angular vibrations of the rotating shaft. An additional moment relative to the center of the ring creates a frictional force on the end contact surface $C$.

Under the action of these forces and moments, the floating ring can vibrate in its plane, as well as perform angular vibrations. Angular vibrations are accompanied by some displacement of the center of gravity of the ring in the axial direction. However, the amplitudes of these displacements are two times less than the amplitudes of the end gap in the joint $C$, which are measured with micrometers even during angular vibrations. Therefore, in the first approximation, axial vibrations can be ignored.

Thus, a self-centering floating ring can be considered as an oscillatory system with four degrees of freedom, performing forced oscillations under the action of kinematic excitation from the shaft. The mass of the ring is usually small compared to the mass of the rotor, so the reverse effect of ring vibrations on rotor vibration can be neglected.

The operation of such a classic design is accompanied by intense radial and angular vibrations. Angular vibrations lead to the formation of periodic, with the shaft rotation frequency, point contacts of the end surfaces. In the case of circular precession of the shaft, the ring motion has the character of a conical precession and the contact patch describes a circular trajectory. Contact stresses increase on the contact patches, which leads to frictional fatigue fractures of the end 
surfaces. There are various constructive methods for suppressing angular vibrations [2], but they require a significant complication of the design and at the same time reduce its reliability.

The behavior of the floating rings is determined by the ratio of forces in the ring and end throttles. The characteristics of the end throttle can vary depending on the contact pressure $p_{c}$. If the contact pressure does not exceed the pressure of the sealed medium $p_{c} \leq p_{1}$, then a liquid friction mode is provided on the end surface. Otherwise, the friction regime becomes boundary and can approach the dry friction regime. In this case, if the self-centering condition is satisfied, the ring vibrations become nonlinear. The third option is that the self-centering condition is not met, the ring loses its radial mobility, and only angular oscillations are possible when the total moment acting on the ring opens the butt joint. Finally, an extreme case, when the systems of forces and moments acting on the ring are balanced, the ring is motionless in the absence of collisions with the shaft.

The last version of working conditions (conditionally movable ring) is the simplest and most reliable.

Let's write down the immobility conditions for the contactless operation mode (no collisions of the shaft with the ring). Radial and angular displacements of the ring can arise under the action of the radial hydrodynamic force $F_{v}$ and the moment $M_{x}$ in the annular gap, as well as under the action of gravity and its moment relative to the point of possible rotation a. Fig. 2 shows the most unfavorable case, when the centering force $F_{y}$ coincides in direction with the force of gravity. The same applies to the moments of these forces.

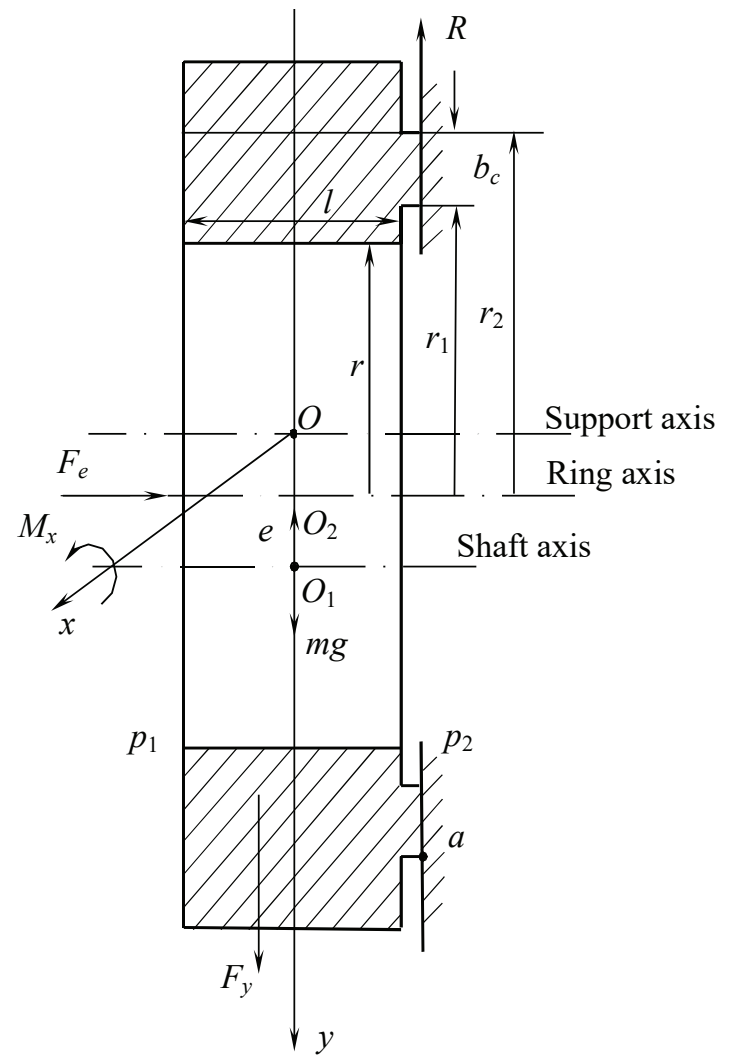

Fig. 2. Design diagram of a conditionally movable ring

Immobility conditions are as follows:

$$
R_{c} \geq F_{y}+m g, \quad 0.5 F_{c}\left(r_{1}+r_{2}\right) \geq M_{x}+0.5 m g l .
$$

The hydrodynamic force and its moment must be calculated for the maximum permissible eccentricity $e_{*} \approx(0.7-0.8) H$ and skew angle $0.5 l \vartheta_{x_{*}} \approx 0.7\left(H-e_{*}\right)$, at which the non-contact operation of the seal can still be guaranteed. In the considered equilibrium position, only the hydrostatic force and moment are preserved 


$$
\begin{gathered}
F_{y}=F_{p y 2}=k_{p}\left[\left(\theta_{0}+N \chi_{m}\right) \varepsilon+(1+2 \Delta \chi) \theta_{x}\right], \\
M_{x}=M_{p x 2}=k_{p} \frac{l}{6}\left(N \Delta \chi \varepsilon+2 \chi_{m} \theta_{x}\right),
\end{gathered}
$$

where

$$
\begin{gathered}
\varepsilon=e / H, \theta_{0}=\vartheta_{0} l / 2 H, \quad \theta_{x}=\vartheta_{x} l / 2 H, \quad \chi_{m}=\chi_{1}+\chi_{2}, \Delta \chi=\chi_{1}-\chi_{2}, \\
\chi_{1}=\varsigma_{11} / \varsigma_{0}, \quad \chi_{2}=\varsigma_{12} / \varsigma_{0}, N=2(1+n) /(2-n), \quad k_{p}=\pi r_{o} \Delta p l / 2 .
\end{gathered}
$$

The constant $n$ characterizes the flow regime ( $n, C$ - exponent and constant in the Blasius formula $\lambda_{0}=C \mathrm{Re}_{0}^{-n}$; laminar flow: $n=1, C=96$, turbulent flow: $n=0.25, C=0.36$, self-similar area: $n=0, C=0.04), \varsigma_{11}, \varsigma_{12}$ - coefficients of local hydraulic losses at the entrance to the annular gap and at the exit from it, $\varsigma_{0}=\left(\varsigma_{11}-\varsigma_{12}\right)+\lambda l / 2 H$.

In general, the force of contact pressure

$$
F_{c}=p_{1} A_{c}+\Delta p\left(A_{e}-A_{c}\right)=\Delta p A_{c}\left(k+p_{2} / \Delta p\right), k=A_{e} / A_{c},
$$

friction force $R_{c}=f F_{c}$. Using expressions for forces and moment, let's reduce relations (1) to the form

$$
\begin{gathered}
k+\frac{p_{2}}{\Delta p} \geq \frac{1}{f \Delta p A_{c}}\left\{k_{p}\left[\left(\theta_{0}+N \chi_{m}\right) \varepsilon+(1+2 \Delta \chi) \theta_{x}\right]+m g\right\}, \\
k+\frac{p_{2}}{\Delta p} \geq \frac{l}{6 f \Delta p A_{c} r_{2}}\left[k_{p}\left(N \Delta \chi \varepsilon+2 \chi_{n} \theta_{x}\right)+3 m g\right] .
\end{gathered}
$$

As a rule, the second condition is weaker, and the fulfillment of the first condition ensures both radial and angular immobility of the seal ring.

Short-term collisions of the ring with the shaft are possible during the start-up period if the ring lies freely on the shaft. Therefore, the cylindrical contact surfaces of the shaft and the ring must be scuff-resistant.

\section{2. Deformable gap seals}

In high-pressure centrifugal machines, noticeable deformations of the elements of gap seals occur under the influence of large pressure drops, regardless of the will of the designer. Since the leakage and dynamic characteristics depend on the shape of the gap, attempts are being made to create such structures, the deformations of which would reduce the leakage and improve the dynamic properties of the annular throttles.

This problem is relatively easy to solve for floating rings [1] (Fig. 3). The ring has a boxshaped cross-section with annular stiffening ribs 1,3 and a thin-walled cylindrical cage 2 , forming a throttling gap with the shaft 4,5 . Fig. 3 shows radial plots of the pressure acting on the ring in the gap 6 and on the outer surface 7. The transverse shading 8 denotes the unbalanced part of the pressure on the outer surface of the ring. The plots are shown for the initial undeformed state of the ring. Under the action of pressure 8, the cylindrical cage bends, acquiring the shape 9 . The change in the shape of the gap entails a change in the pressure distribution in it 10 and a decrease in the external load 8 . The equilibrium deformed state of the ring is determined by solving the static problem of hydroelasticity.

The deformation of the ring leads to a decrease in the flow area of the annular gap and to a decrease in leakage. It is also important that deformations grow with increasing compacted pressure, and this, in turn, slows down the growth of leaks. Contractor shape 9 of the main part of the gap increases its stiffness and damping properties.

It is more difficult to provide the required deformation of the front gap seals of the impellers. A relatively simple solution [8] is shown in Fig. 4. Sleeve 1 with a thin-walled cylindrical cage 2 forms a cavity A with the body, connected by holes 3 with a throttling annular gap 4 . In the undeformed state of cage 2 , the pressure along the length of the gap 4 varies linearly from the maximum 
at the inlet to the minimum at the outlet. The pressure in cavity $A$ is equal to the pressure in the place of the gap 4, where the holes 3 . Thus, the radial pressure drop acts on the cage, increasing towards the exit from the gap 4. The radial deformations of the sleeve 1 under the action of this difference lead to an increase in the pressure in the gap 4 and, respectively, in cavity $A$. As a result, the pressure drop that deforms the sleeve decreases. The deformation effect is the same as in the floating ring.

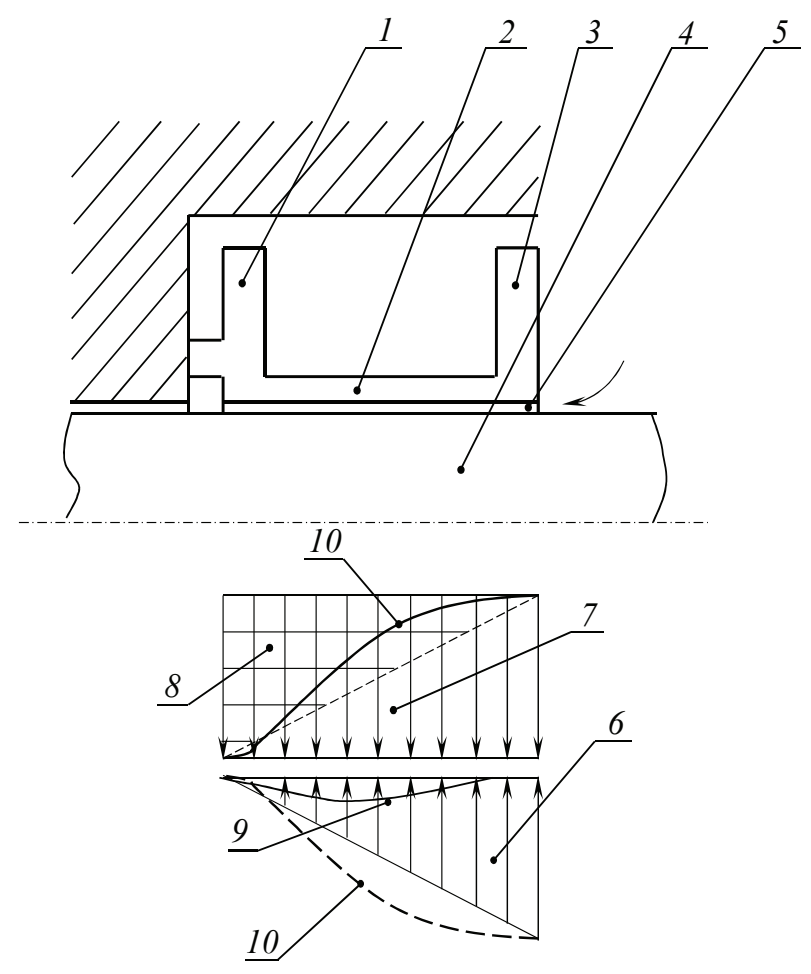

Fig. 3. Deformable floating ring and pressure curves

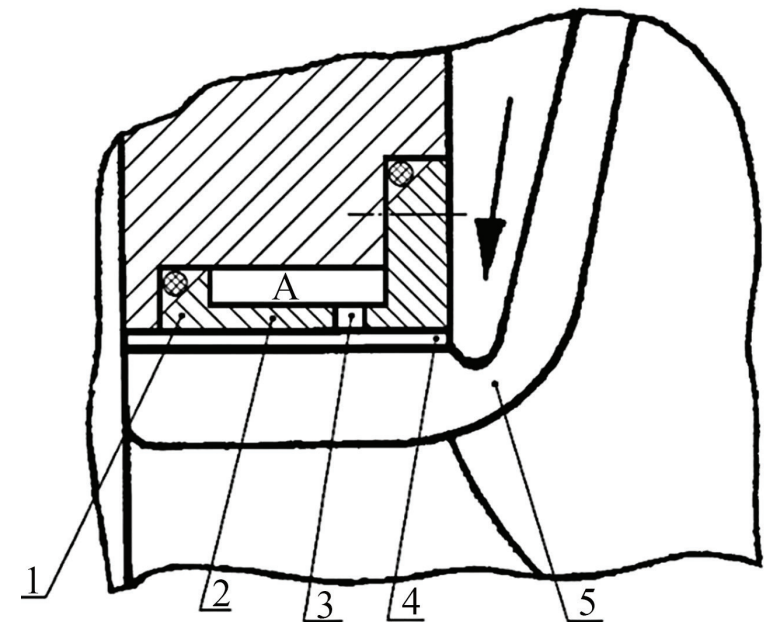

Fig. 4. Deformable impeller gap seal

Another design of the gap seal [9] is shown in Fig. 5. Axially movable deformable sleeve 3 with an annular shoulder 5 forms a cavity A in the housing 1, connected to the throttling gap 6 by holes 4 . The pressure in the cavity $\mathrm{A}$ is constant along the length, and in the gap 6 decreases along the length.

In the equilibrium state, the axial pressure force in the cavity $A$ together with the force of the spring 2 and the friction force balances the axial force of pressure on the end face 7. An increase 
in the sealing pressure shifts the sleeve to the left. In this case, the sleeve is additionally deformed, the clearance at the outlet decreases, and the pressure in cavity A increases. The movement of the sleeve stops after the restoration of the conditions of its axial equilibrium.

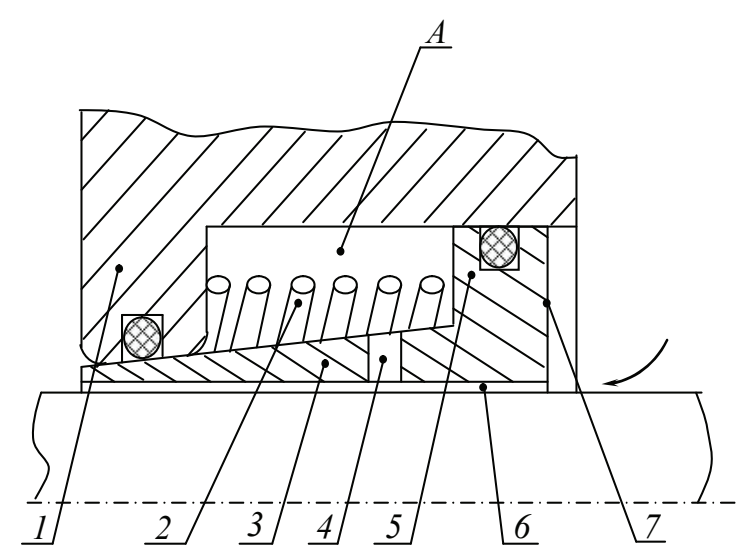

Fig. 5. Seal with deformable axially movable sleeve

\section{3. Labyrinth seals}

The problem of ensuring the contactless operation of gap seals is especially relevant for pumps that pump flammable liquids, and for compressors that pump low-viscosity gases that do not have lubricating properties. There is a risk of fire in pumps, in compressors there is a risk of the rotor getting stuck in the seals.

To seal gaseous media in centrifugal compressors, in steam and gas turbines, various designs of labyrinth seals with minimal contact surfaces are used [10] (Fig. 6). The annular ridges in these seals are made of metal tape $0.15-0.2 \mathrm{~mm}$ thick. With such a thickness, possible contacts only crush the ridges, without causing excessive heating, enveloping and seizing.
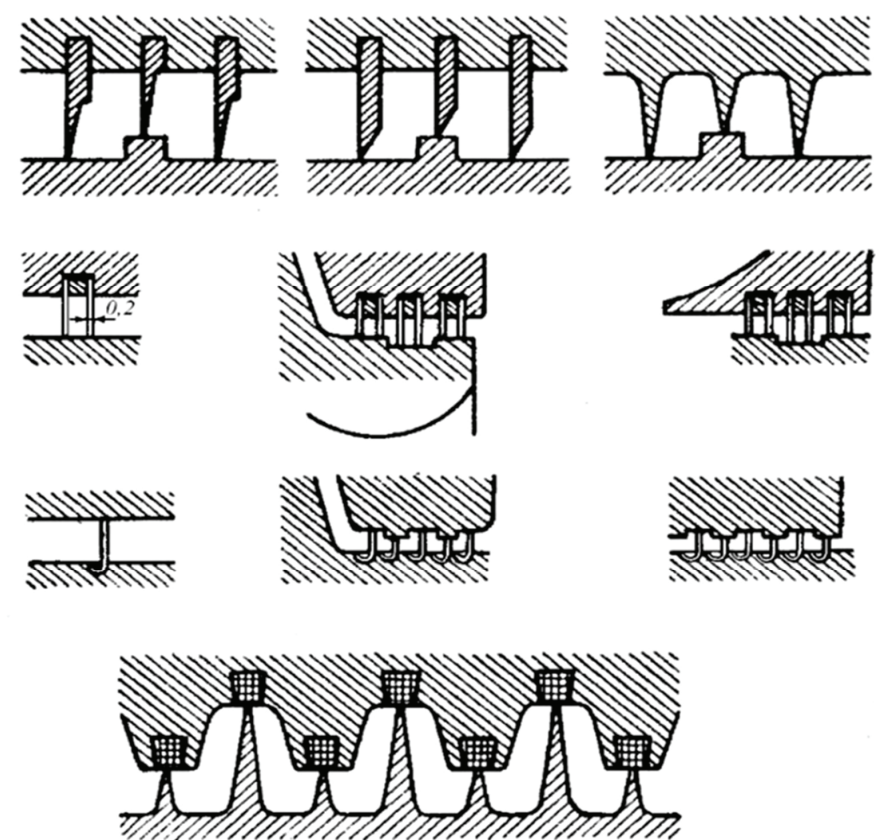

Fig. 6. Some designs of labyrinth seals for gas

The disadvantage of gas labyrinth seals is the low flexural rigidity of the ridges. This drawback is eliminated in special labyrinth seals, called honeycomb seals by the type of cellular structure of the sealing element [11] (Fig. 7). The seal is a sleeve, inside of which a honeycomb filler is fixed by high-temperature vacuum brazing with powder solders. It has the form of hexagonal cells 
and is made of corrugated strips of steel corrosion-resistant foil, connected along the contacting edges by contact welding on automatic machines. Such a honeycomb structure has increased rigidity and does not crumple under high pressure drops. The depth of the cells is $4-6 \mathrm{~mm}$, the facet size is $2-4 \mathrm{~mm}$, and the foil thickness is $0.05-0.15 \mathrm{~mm}$. The thinner thickness and improved heat dissipation mitigate the harmful effects of scuffing, allowing seals to be made with almost no radial clearance, thereby minimizing leakage of the sealed medium. The honeycomb cage is used in combination with a smooth shaft or a shaft with labyrinth ridges. The latter combination is used to seal the stages of gas turbine engines. In case of misalignment, the ridges located on the rotor easily cut into the honeycomb walls, causing their slight deformations and local heating. The gas flow rate remains almost unchanged, while if the ridges of a conventional labyrinth seal are damaged, the leaks increase significantly.

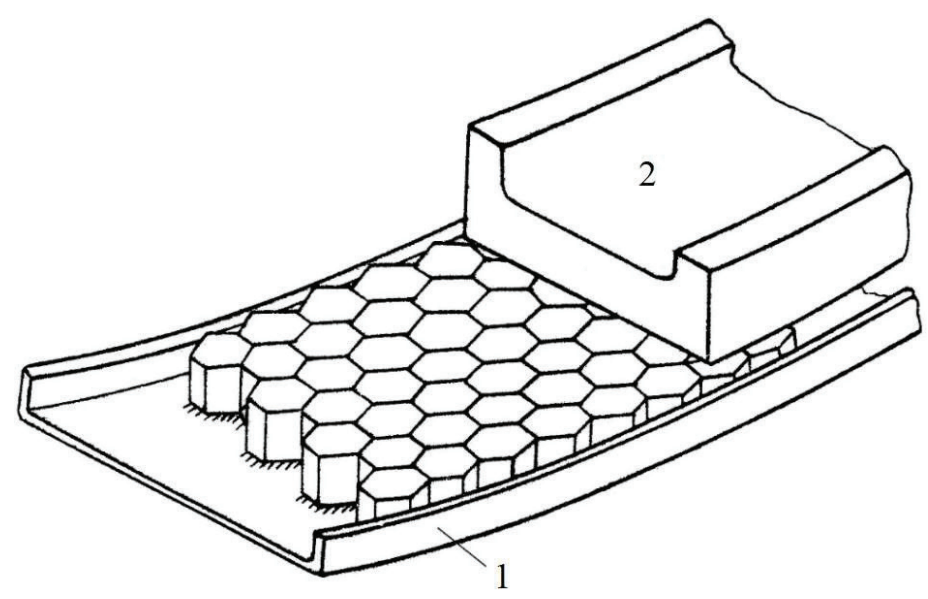

Fig. 7. Honeycomb seal

To reduce the risk of contacts between the rotating and stationary elements of the seals, the pumps use labyrinth seals with increased radial clearances up to $0.5 \mathrm{~mm}$. To limit excessive leakage growth, seals are made with overlapping ridges (Fig. 8).
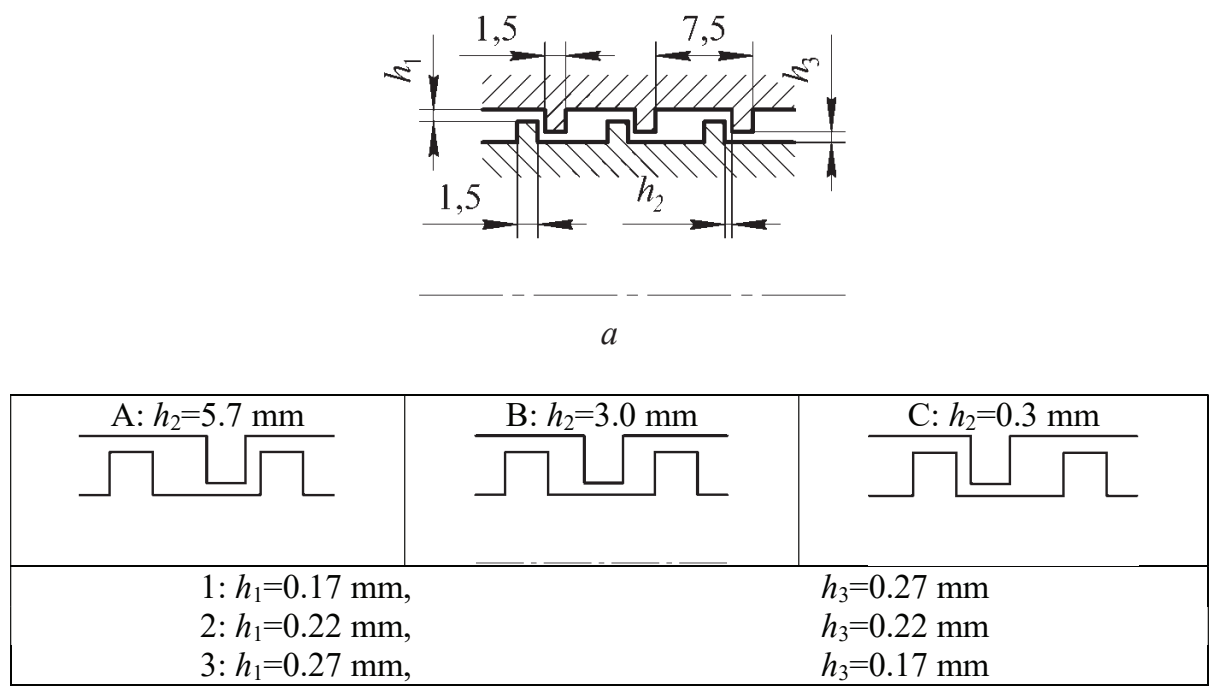

$b$

Fig. 8. Labyrinth seal with overlapping ridges: $a$ - design diagram; $b$ - versions

The dynamic properties of such seals substantially depend on the relative position of the ridges. Option C1 (Fig. 8) caused self-excited vibrations even of a non-rotating shaft. In this regard, as an alternative, it was proposed [12] construction of a well seal (Fig. 9). The well seal is a sleeve 1, 
on the inner surface of which sickle-shaped wells are milled 2. In the axial direction, the rows of wells are separated by ridges 4 , and in the circumferential direction, adjacent wells are separated by bridges 3 . The ridges perform the same role as ridges in conventional labyrinth seals, and the bridges inhibit the circumferential flow.
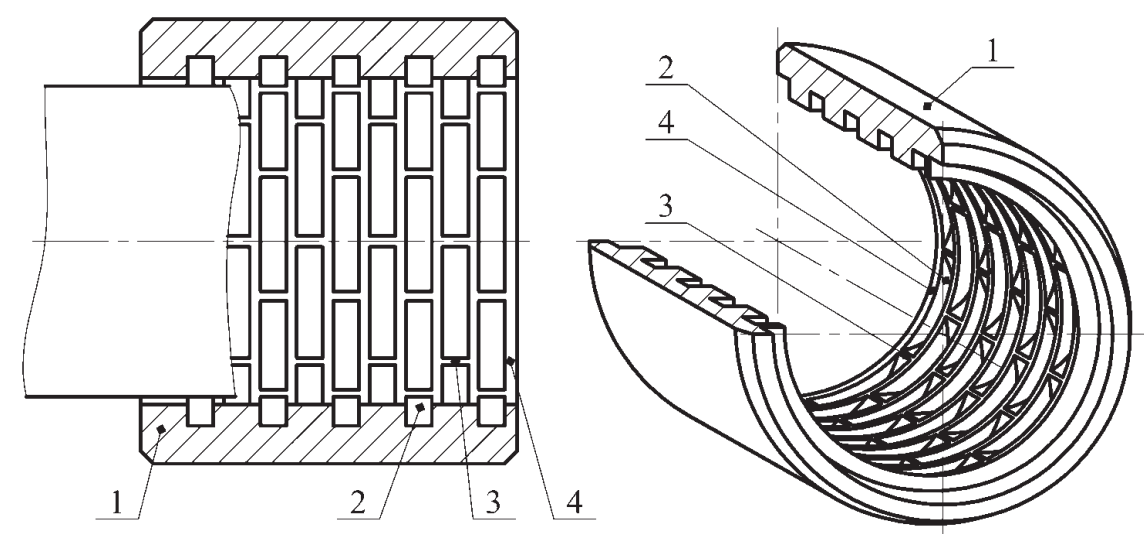

Fig. 9. Well seal

The role of the bridges is especially important, since, by slowing down the circumferential flow, they thereby reduce the circulating force, which is the main reason for the loss of dynamic stability of the rotor in the seals. In addition, the holes are semi-closed chambers that inhibit the displacement flow and increase the damping force, respectively.

A significant influence on the rigidity of labyrinth seals is exerted by the type of relative position of the ridges. The maximum rigidity is possessed by the A3 variant (Fig. 8), in which the channel resembles a contractor shape. Diffuser flow seals (option $\mathrm{C} 1$ ) can have negative stiffness. When testing this version at pressure drops greater than $0.5 \mathrm{MPa}$, the sleeve of the non-rotating rotor was pressed against the stator yoke, i.e. lost static stability. A further increase in the pressure drop to $4 \mathrm{MPa}$ led to the emergence of intense self-excited oscillations, the amplitudes of which reached the value of the radial gap.

\section{Discussion}

The works of the creators of centrifugal machines [1-6] was devoted to the study of the relationship between the vibration characteristics of the rotor and the designs of gap seals. These works, as well as the needs of thermal and nuclear energy in feed, main circulation and other pumps for ever higher parameters stimulated a detailed study of the problems of hydrodynamics of gap seals and their effect on the vibration state of rotors of centrifugal machines $[1,2,5,6]$. Work on the creation of turbopump assemblies for high-power rocket engines for reusable spacecraft further raised the interest of researchers in the dynamic characteristics of gap seals and vibrations of rotors in seals $[7,8,10]$.

The results obtained in this work in many aspects confirm the qualitative picture of the physical processes occurring in various designs of gap seals, described by other researchers.

The peculiarity of the works of most researchers of the influence of seals on the dynamics of a centrifugal machine is that the rotor and seals are considered as a closed hydromechanical system, in which the role of a connecting link is played by the flow of the pumped medium in throttling channels, inertial and hydrodynamic characteristics of the flow [3-6].

The positive qualities of gap seals are realized provided that the non-contact mode of operation is maintained, when there is no danger of scoring. And for this it is necessary that the initial clearance value (for radial seals) exceeds the total eccentricity of the shaft relative to the sealing ring. Eccentricity can appear due to manufacturing and assembly errors (runout, radial and angular misalignment), as a result of force and temperature deformations, and also due to shaft vibrations. For these reasons, the gap has to be assigned in the range of at least $0.2-0.4 \mathrm{~mm}$. Therefore, the leakage through gap seals of new centrifugal pumps reaches $5 \%$ of the pump flow. During opera- 
tion, leaks can increase significantly due to erosional erosion of the sealing gaps, and this leads to a corresponding decrease in the efficiency.

A conditionally movable floating ring works as a stationary gap seal, and the hydrodynamic forces arising in the annular gap are completely transferred to the rotor, predetermining its vibration state. Dynamic problems, which are of prime importance for floating rings, are eliminated in this case. Due to the correct choice of the shape of the gap, it is possible to increase the coefficients of hydrostatic stiffness and damping and thereby reduce the amplitudes of lateral vibrations of the rotor. Considering that shaft vibrations in the area of the seals can be several times greater than on bearings, then even relatively small radial clearances of $0.05-0.1 \mathrm{~mm}$ ensure non-contact operation of semi-movable floating rings. Thus, seals with conditionally movable rings make it possible, under certain conditions, to exclude collisions between the shaft and the ring even with small (less than $0.1 \mathrm{~mm}$ ) gaps between them. The results obtained in some aspects are confirmed by the results published in [7].

All the described designs of deformable impeller seals require careful manufacturing and fine-tuning, and their optimal design requires solving the corresponding problems of hydroelasticity. Therefore, it is not economically feasible to use them for ordinary serial machines, and for unique machines with high requirements for tightness and vibration reliability, such seals can be indispensable. An example is the turbopump units of rocket engines, the power of which is measured in hundreds of megawatts at extremely low mass and dimensional characteristics. The efficiency of using deformable gap seals in the design of turbopump units of rocket engines was confirmed in [8].

The dynamic properties of labyrinth seals substantially depend on the relative position of the ridges. The results of the study of labyrinth seals presented in this work in a number of fundamental nuances developed and supplemented the studies [10, 11].

Labyrinth seals with overlapping ridges have extremely low radial stiffness, which is primarily due to the free circumferential fluid flow through relatively large channels between the ridges. As a result, the circumferential pressure is equalized and the radial force is reduced. This remark applies to both straight-through labyrinth seals and screw seals. Thus, labyrinth seals with annular grooves represent a potential hazard to high-pressure pumps and compressors. The test results of the labyrinth seals of a high-pressure compressor, confirming the research results, are given in [12].

If vibration and leakage mitigation is equally important for a particular pump, well seals should be used. In terms of hydraulic resistance, they are not inferior to labyrinth and honeycomb ones, and in terms of elastic damping properties they significantly surpass them. At the same time, they are less prone to scuffing and seizure than conventional O-ring gap and labyrinth seals. It should be borne in mind that the coefficient of hydrostatic stiffness of well seals is approximately two times less than that of conventional gap seals.

\section{Conclusions}

Floating ring seals are often more effective when the rings are not floatable.

Deformable impeller seals are advisable to be used for unique machines with high requirements for tightness and vibration reliability.

Of the labyrinth seal designs described, overlapping ridge seals have the worst dynamic performance. The honeycomb seal has more favorable dynamic properties, and the well seal has the best combination of consumption and dynamic characteristics.

\section{References}

[1] Martsinkovskii, V. A., Shevchenko, S. S.; Shevchenko, S. S. (Ed.) (2018). Nasosy atomnykh elektrostantsii: raschet, konstruirovanie, ekspluatatsiia. Sumy: Universitetskaia kniga, 472.

[2] Martsynkovskyi, V. A. (1980). Beskontaktnie uplotnenyia rotornikh mashyn. Moscow: Mashynostroenye, 200.

[3] Kim, S. H., Ha, T. W. (2016). Prediction of leakage and rotordynamic coefficients for the circumferential-groove-pump seal using CFD analysis. Journal of Mechanical Science and Technology, 30 (5), 2037-2043. doi: http://doi.org/10.1007/s12206$016-0410-4$ 
[4] Vijaykumar, A., Morrison, G. (2010). Numerical Simulation of the Flow Field in a Statically and Dynamically Eccentric Annular Seal With Non-Circular Whirl Orbits. ASME 2010 3rd Joint US-European Fluids Engineering Summer Meeting: Volume 1, Symposia - Parts A, B, and C, 731-761. doi: http://doi.org/10.1115/fedsm-icnmm2010-30839

[5] Wang, J., Shen, H. P., Ye, X. Y., Hu, J. N., Feng, Y. N. (2013). The influence of annular seal clearance to the critical speed of the multistage pump. IOP Conference Series: Materials Science and Engineering, 52 (2), 022034. doi: http://doi.org/10.1088/1757$899 \mathrm{x} / 52 / 2 / 022034$

[6] Bojko, M., Kozubkova, M., Kozdera, M., Zavila, O. (2014). Investigation of the influence of radial grooves on the flow in an eccentrically deposited annulus using CFD numerical simulation. EPJ Web of Conferences, 67, 02009. doi: http://doi.org/10.1051/ epjconf/20146702009

[7] Badun, O. P., Deshevykh, S. A., Ivanov, Ia. N. (2016). Osobennosti ispolzovaniia plavaiuschikh kolets v kislorodnykh nasosakh. Vestnik dvigatelestroeniia, 2, 115-121.

[8] Suleimanov, R. I., Nazarov, V. P. (2011). Analiz vozmozhnosti primeneniia schelevogo uplotneniia rotora TNA s deformiruemoi vtulkoi. Aktualnye problemy aviatsii i kosmonavtiki, 7, 66-67.

[9] Martsinkovskii, V. A., Gulii, A. N., Melnik, V. A. (1988). A. s. 1372138. Uplotnenie vala.

[10] Makarov, A., Zaytsev, N. (2015). Engineering and theoretical problems of labyrinth seals application in high-speed rotor machines. Perm National Research Polytechnic University Aerospace Engineering Bulletin, 42, 61-81. doi: http://doi.org/ 10.15593/2224-9982/2015.42.05

[11] GAO, R. (2012). Computational Fluid Dynamic and Rotordynamic Study on the Labyrinth Seal. Ph.D. Dissertation in Mechanical Enginee-ring. Virginia Polytechnic Institute and State University.

[12] Martsinkovskii, V. S., Kukharev, I. E., Bilyk, Ia. I., Lazarenko, A. D. (2016). Resheniia problem germetizatsii protochnogo trakta tsentrobezhnogo kompressora. Kompressornoe i energeticheskoe mashinostroenie, 4, 5-14. 